\title{
Ácido Ascórbico e Eletroterapia - Terapia Combinada no Tratamento do Melasma: Uma Revisão da Literatura
}

\author{
Adriana da Silval ; Livia Mara Gomes Pinheiro ${ }^{2}$
}

\begin{abstract}
Resumo: O melasma é uma das disfunções estéticas faciais que mais acomete as mulheres em idade reprodutiva, é uma hipermelanose adquirida, caracterizada por manchas escuras ou acastanhadas. Ocorre com maior frequência nas regiões malar, centrofacial e mandibula. A utilização da eletroterapia associada ao ácido ascórbico (vitamina C) é a nova proposta para controle do melasma. Neste contexto o referido artigo tem como principal objetivo analisar os efeitos do ácido ascórbico e eletroterapia no tratamento do melasma. Esta pesquisa configura-se como uma revisão integrativa de literatura, realizada no período de janeiro a julho de 2017, baseada em artigos extraídos das bases de dados do Google acadêmico, Scielo, Lilacs e Madline, usando como palavras chaves: Hipercromias. Melasma. Ácido Ascórbico. Vitamina C. Eletroterapia. Como resultados obtidos no levantamento de informações contidas nos artigos consultados podemos concluir que, as terapias menos agressivas no controle do melasma podem demandar maior tempo para se obter os resultados em relação aos tratamentos mais agressivos, como é o caso do uso da Vitamina $\mathrm{C}$ associada a Eletrterapia.
\end{abstract}

Palavras-chave: Hipercromias. Melasma. Ácido Ascórbico. Vitamina C. Eletroterapia.

\section{Ascorbic Acid and Electrotherapy - Combination Therapy in Melasma Treatment: A Literature Review}

\begin{abstract}
Absctrat: The melasma is one of the facial aesthetic disorders that more affects women of reproductive age, is an acquired hypermelanosis, characterized by dark spots or brown in colour. Occurs more often in regions centrofacial mandibular and malar, (SANCHEZ et al, 1981). The use of electrotherapy associated with vitamin $\mathrm{C}$ is the new proposal for control of melasma. In this context that article has as main objective to analyze the effects of Ascorbic acid and electrotherapy in the treatment of melasma. This research appears as an integrative literature review, carried out in the period January to July 2017, based on articles extracted from the Google Scholar databases, Scielo, Lilacs and Madline, using key words: Hipercromias. Melasma. Ascorbic Acid. Electrotherapy. As results obtained in the survey of information contained in the articles consulted we can conclude that the less aggressive therapy in the control of melasma can demand longer to get the results.
\end{abstract}

Keyword: Hipercromias. Melasma. Ascorbic Acid. Electrotherapy.

\footnotetext{
${ }^{1}$ Curso Tecnólogo em Estética e Cosmética da Faculdade Independente do Nordeste. Contato: didi_ca_dr@ hotmail.com;

2 Graduação em Bacharel em Enfermagem pela Universidade Estadual do Sudoeste da Bahia- Campus Jequie. Mestrado em Ética e Gestão, Pós graduada em Saúde Pública, Urgência e Emergência, Saúde Mental, Ensino na área de Saúde e Dermato Funcional. Atualmente é professora da Faculdade Independente do Nordeste. Contato: liviamara@ fainor.com.br.
} 


\section{Introdução}

O melasma é uma das disfunções estéticas faciais que mais acomete as mulheres em idade reprodutiva, é uma hipermelanose adquirida, caracterizada por manchas escuras ou acastanhadas. Ocorre com maior frequência nas regiões malar, centrofacial e mandibular (SANCHEZ et. al, 1981).

De acordo com (RIBEIRO, 2010) o melasma é caracterizado por manchas claras ou escuras, de bordas irregulares, reticulares e simétricas, que aparecem mais na face, abrangendo a região malar, fronte, lábios superiores e queixo. Acomete todos os fototipos de pele, principalmente os mais elevados.

Por acometer regiões foto expostas, causa um impacto negativo na qualidade de vida das pessoas, tanto socialmente como emocionalmente, comprometendo sua autoestima, por isso seu tratamento é muito importante. (MATOS; CAVALCANTI, 2009)

Seu diagnóstico é baseado na profundidade do pigmento melânico, podendo o exame ser realizado pela lâmpada de wood, definindo o melasma em epidérmico, dérmico, misto ou indefinido, sendo o mais comum o epidérmico, pois a deposição de melanina é maior nas camadas basal e supra basal (MATOS; CAVALCANTI,2009)

Umas das causas para o surgimento e piora do melasma é a radiação ultravioleta (UV), pois a mesma interfere diretamente na melanogênese, desencadeando assim uma hiperpigmentação epidérmica de difícil controle. Os hormônios sexuais como estrogênio e progesterona também estão relacionados ao surgimento do melasma.

A terapia despigmentante tem como objetivo clarear e melhorar o aspecto da mancha e o resultado irá depender da localização e da deposição do pigmento, pois quanto mais superficial estiver mais rápido terá um resultado satisfatório.

As terapias de reposição hormonal e a gravidez também são fatores desencadeantes para o surgimento do melasma, devido ao aumento da progesterona e estrogênio (WV IB et al, 2012).

De acordo com (LAKHDAR et al, 2007) o melasma tente a melhorar no inverno e piorar muito no verão (ou exposição intensa ao sol). O uso de protetor solar de alta proteção reduz até metade o aparecimento da discromia e reduz sua incidência em até $90 \%$ na gestação.

O tratamento tem como objetivo clarear e melhorar o aspecto da mancha e o resultado irá depender da localização da deposição de pigmento, pois quanto mais superficial estiver mais rápido terá um resultado. A utilização de recursos eletroterápicos associados a vitamina $\mathrm{C}$ foi 
proposto para o presente estudo por possuírem ações como: ativação da circulação sanguínea local, despigmentantes, antioxidantes e reepitelizadores da epiderme.

Este estudo se justifica pela necessidade de sistematizar através de artigos científicos os efeitos dos tratamentos propostos para controle do melasma com técnicas menos agressivas e comparar os resultados do uso de cada um dos itens propostos na investigação, bem como conhecer a eficácia dos mesmos no clareamento do melasma.

\section{Objetivos}

O melasma é caracterizado por manchas claras ou escuras, de bordas irregulares, reticulares e simétricas, que aparecem mais na face, abrangendo a região malar, fronte, lábios superiores e queixo. Acomete todos os tipos de pele, principalmente os mais elevados (RIBEIRO, 2010).

Este artigo tem como principal objetivo, avaliar através de revisão de literatura os efeitos do ácido ascórbico (vitamina C) e eletroterapia no tratamento do melasma com métodos menos agressivos. De acordo com os artigos analisados, o melasma é uma disfunção estética de difícil cura porem controlável.

\section{Justificativa}

Um estudo como este é importante pois foi possível observar, através da revisão dos artigos que o melasma é uma disfunção estética multifatorial, ou seja, de causa desconhecida. Por acometer regiões foto expostas, causa um impacto negativo na qualidade de vida das pessoas, tanto socialmente como emocionalmente, comprometendo sua autoestima, por isso seu tratamento é muito importante. (MATOS; CAVALCANTI, 2009.

A terapia despigmentante tem por finalidade clarear e melhorar o aspecto da mancha e o resultado irá depender da localização e da deposição do pigmento, pois quanto mais superficial estiver mais rápido terá um resultado satisfatório. Este estudo se justifica pela necessidade de analisar a eficácia dos tratamentos propostos para controle do melasma com técnicas menos agressivas e de fácil aplicação. 
A escolha do tema abordado decorre do fato da terapia por microcorrente ser uma alternativa de tratamento da revitalização cutânea e do clareamento da pele através de seus efeitos fisiológicos, tendo em vista que as discromias não apresentarem um tratamento específico. O presente artigo tem por objetivo: analisar e discutir os efeitos da microcorrente associada a vitamina $\mathrm{C}$ na hiperpigmentação facial.

\section{Metodologia}

Esta pesquisa configura-se como uma revisão bibliográfica que representa esclarecimentos científicos, por meio de conhecimentos conceituados, acessíveis a partir de uma temática. Este contribui para o pesquisador adquirir informações para sua pesquisa e propor seu ponto de vista de acordo com o que foi descrito pelo autor, identificando as teorias fundamentadas, averiguando, comentando e abordando segundo sua compreensão de forma explicativa e parafraseada do problema, tendo como fonte a investigação dada no texto pelo autor.

De acordo com Cook et al. (1997) a revisão bibliográfica pode ser narrativa ou sistemática. O primeiro tipo é baseado em uma descrição simplificada de estudos e informações sobre um determinado assunto. O segundo tipo, apesar de também ter o caráter narrativo, é baseado na aplicação de métodos com maior rigor científico, podendo alcançar melhores resultados.

Esse processo permite ao pesquisador compilar dados e refinar hipóteses através de uma pesquisa minuciosa baseada em artigos extraídos das bases de dados do Google acadêmico, Scielo, Lilacs e Madline. Como critérios de inclusão foram utilizados artigos cuja temática abordasse o tema proposto e por meio de palavras chave encontradas nos títulos e resumos, e obras com publicações compreendidas no período de 2007 a 2017, em português e em inglês, e que abordassem o tema mencionado. Como critérios de exclusão foram retirados estudos que não se enquadraram no período. 


\section{Resultados e Discussões}

$\mathrm{Na}$ sequência, a tabela onde constam os artigos utilizados para análise na presente revisão.

Tabela 1 - Artigos utilizados na revisão

\begin{tabular}{|c|c|c|c|c|c|c|c|}
\hline $\mathrm{N}$ & $\begin{array}{l}\text { TITULO DO } \\
\text { ARTIGO }\end{array}$ & AUTOR & $\begin{array}{l}\text { ANO DE } \\
\text { PUBLI- } \\
\text { CAÇÃO }\end{array}$ & $\begin{array}{l}\text { CATE- } \\
\text { GORIA }\end{array}$ & SUPORTE & $\begin{array}{c}\text { TIPO } \\
\text { DA } \\
\text { PESQUISA }\end{array}$ & OBJETIVOS \\
\hline 1 & $\begin{array}{c}\text { Ingredientes } \\
\text { naturais são } \\
\text { eficazes na gestão } \\
\text { da } \\
\text { hiperpigmentação? } \\
\text { Uma revisão } \\
\text { sistemática. }\end{array}$ & $\begin{array}{l}\text { HOLLINGER, } \\
\text { et al. }\end{array}$ & 2018 & Artigo & $\begin{array}{l}\text { J Clin Aesthet } \\
\text { Dermatol. }\end{array}$ & $\begin{array}{l}\text { Estudo de } \\
\text { campo }\end{array}$ & $\begin{array}{c}\text { Revisão de estudos } \\
\text { clínicos avaliando } \\
\text { o uso de diferentes } \\
\text { produtos naturais } \\
\text { no tratamento de } \\
\text { hiperpigmentação, } \\
\text { tendo o ácido } \\
\text { ascórbico como um } \\
\text { desses ingredientes. }\end{array}$ \\
\hline 2 & $\begin{array}{l}\text { Melasma: uma } \\
\text { revisão abrangente } \\
\text { e atualizada. }\end{array}$ & $\begin{array}{l}\text { HASSUN, et } \\
\text { al. }\end{array}$ & 2017 & Artigo & $\begin{array}{l}\text { RBM: revista } \\
\text { brasileira de } \\
\text { medicina }\end{array}$ & Revisão & $\begin{array}{c}\text { Esta revisão serve } \\
\text { como uma } \\
\text { atualização } \\
\text { abrangente sobre a } \\
\text { compreensão atual } \\
\text { da epidemiologia, } \\
\text { patogênese, } \\
\text { características } \\
\text { clínicas e } \\
\text { histológicas do } \\
\text { melasma. }\end{array}$ \\
\hline 3 & $\begin{array}{l}\text { Melasma: uma } \\
\text { revisão clínica e } \\
\text { epidemiológica }\end{array}$ & $\begin{array}{l}\text { BOLANCA, } \\
\text { et al }\end{array}$ & 2014 & Artigo & $\begin{array}{c}\text { Anais } \\
\text { Brasileiro de } \\
\text { Dermatologia }\end{array}$ & $\begin{array}{l}\text { Estudo de } \\
\text { Campo }\end{array}$ & $\begin{array}{c}\text { Argumenta sobre } \\
\text { os os principais } \\
\text { aspectos clínicos e } \\
\text { epidemiológicos do } \\
\text { melasma. } \\
\end{array}$ \\
\hline 4 & $\begin{array}{l}\text { Aggravating factors } \\
\text { for melasma: a } \\
\text { prospective study in } \\
197 \text { Tunisian } \\
\text { patients. }\end{array}$ & $\begin{array}{l}\text { GUINOT C, et } \\
\text { al. }\end{array}$ & 2010 & Artigo & $\begin{array}{l}\text { J Eur Acad } \\
\text { Dermatol } \\
\text { Venereol. }\end{array}$ & $\begin{array}{c}\text { Estudo } \\
\text { prospectivo }\end{array}$ & $\begin{array}{l}\text { Investigar fatores } \\
\text { que influenciam a } \\
\text { gravidade do } \\
\text { melasma em uma } \\
\text { grande população. }\end{array}$ \\
\hline 5 & $\begin{array}{c}\text { Clinico- } \\
\text { epidemiological } \\
\text { Study of Patients } \\
\text { with } \\
\text { Melasma in a } \\
\text { Tertiary Care } \\
\text { Hospital - A } \\
\text { Prospective } \\
\text { Study }\end{array}$ & WV IB, et al & 2017 & Artigo & $\begin{array}{l}\text { International } \\
\text { Journal of } \\
\text { Scientific } \\
\text { Study }\end{array}$ & $\begin{array}{l}\text { Estudo de } \\
\text { Campo }\end{array}$ & $\begin{array}{c}\text { Estabelecer o } \\
\text { padrão } \\
\text { epidemiológico do } \\
\text { melasma. }\end{array}$ \\
\hline 6 & $\begin{array}{c}\text { Melasma, um } \\
\text { distúrbio do } \\
\text { fotoenvelhecimento. }\end{array}$ & $\begin{array}{c}\text { PASSERON } \\
\mathrm{T} \text {, et al. }\end{array}$ & 2017 & Artigo & $\begin{array}{l}\text { the official } \\
\text { journal } \\
\text { ofinternational } \\
\text { federation of } \\
\text { pigment cell } \\
\text { societies · } \\
\text { society for } \\
\text { melanoma } \\
\text { research }\end{array}$ & $\begin{array}{l}\text { Estudo de } \\
\quad \text { caso }\end{array}$ & $\begin{array}{c}\text { Evidências mais } \\
\text { recentes sobre a } \\
\text { fisiopatologia do } \\
\text { melasma e } \\
\text { sugerimos que o } \\
\text { melasma pode ser } \\
\text { um distúrbio } \\
\text { cutâneo do } \\
\text { fotoenvelhecimento } \\
\text { que afeta } \\
\text { indivíduos } \\
\text { geneticamente } \\
\text { predispostos. }\end{array}$ \\
\hline 7 & $\begin{array}{l}\text { What should be } \\
\text { considered in }\end{array}$ & $\begin{array}{l}\text { KANG HY,et } \\
\text { al }\end{array}$ & 2010 & Artigo & $\begin{array}{c}\text { Ann } \\
\text { Dermatol. } \\
\text { 2010 Nov }\end{array}$ & $\begin{array}{c}\text { Estudo } \\
\text { Randomizado }\end{array}$ & $\begin{array}{l}\text { O que deve ser } \\
\text { considerado no }\end{array}$ \\
\hline
\end{tabular}




\begin{tabular}{|c|c|c|c|c|c|c|c|}
\hline & $\begin{array}{l}\text { treatment of } \\
\text { melasma. }\end{array}$ & & & & $\begin{array}{l}22 \text { (4): 373- } \\
\text { 378. Inglês. }\end{array}$ & & $\begin{array}{c}\text { diagnóstico de } \\
\text { melasma para que } \\
\text { o tratamento tenha } \\
\text { êxito. }\end{array}$ \\
\hline 8 & $\begin{array}{c}\text { Patologia } \\
\text { Heterogênea do } \\
\text { Melasma e suas } \\
\text { Implicações } \\
\text { Clínicas }\end{array}$ & $\begin{array}{l}\text { KWON SH, et } \\
\text { al. }\end{array}$ & 2016 & Artigo & $\begin{array}{l}\text { Int J Mol Sci. } \\
2016\end{array}$ & $\begin{array}{c}\text { Revisão } \\
\text { Bibliográfica }\end{array}$ & $\begin{array}{c}\text { Esta revisão discute } \\
\text { essas } \\
\text { características } \\
\text { histológicas do } \\
\text { melasma, levando } \\
\text { em consideração } \\
\text { suas implicações } \\
\text { no tratamento do } \\
\text { melasma. }\end{array}$ \\
\hline 9 & $\begin{array}{c}\text { Avanços no } \\
\text { tratamento do } \\
\text { melasma: uma } \\
\text { revisão da literatura } \\
\text { recente. }\end{array}$ & $\begin{array}{l}\text { BOLA } \\
\text { AREFIEV } \\
\text { KL, et al. }\end{array}$ & 2012 & Artigo & $\begin{array}{c}\text { Dermatol } \\
\text { Surg. 2012; }\end{array}$ & $\begin{array}{c}\text { Revisão } \\
\text { Bibliográfica }\end{array}$ & $\begin{array}{c}\text { Revisar os recentes } \\
\text { avanços no } \\
\text { tratamento do } \\
\text { melasma. }\end{array}$ \\
\hline 10 & $\begin{array}{l}\text { Tratamento do } \\
\text { melasma. }\end{array}$ & $\begin{array}{l}\text { RENDON M, } \\
\text { et al. }\end{array}$ & 2006 & Artigo & $\begin{array}{l}\text { J Am Acad } \\
\text { Dermatol. }\end{array}$ & $\begin{array}{c}\text { Revisão } \\
\text { Bibliográfica }\end{array}$ & $\begin{array}{l}\text { Avaliar a eficácia } \\
\text { clínica dos } \\
\text { diferentes } \\
\text { tratamentos do } \\
\text { melasma, a fim de } \\
\text { gerar uma } \\
\text { declaração } \\
\text { consensual sobre a } \\
\text { sua gestão }\end{array}$ \\
\hline
\end{tabular}

Fonte: Autora da Pesquisa.

Os elementos desta pesquisa foram adquiridos através de revisões bibliográficas, foi por meio da análise de artigos científicos que surgiu a necessidade de abordar o tema: "Ácido Ascórbico (vitamina C) e Eletroterapia - terapia combinada no tratamento do melasma".

O termo melasma origina-se da palavra "melas" que significa preto, refere-se ainda a sua apresentação acastanhada (BOLANCA et al, 2008)

O melasma é uma disfunção estética que acomete especialmente as mulheres, é um distúrbio na melanogênese que resulta em uma hiperpigmentação localizada (HANDEL, et al, 2014)

De acordo com (SANCHEZ et al, 1981) o melasma é uma hipermelanose comum, adquirida, simétrica de contorno irregular, porém com limites nítidos. Caracteriza-se por máculas acastanhadas, de intensidades diferentes, em áreas fotoexpostas, especialmente na face, fronte e têmporas, e mais raramente no nariz, pálpebras e mento.

Segundo Sheth (2011) o melasma é a melanodermia mais comum em indivíduos com pele marrom a clara que afeta a população fortemente melanizada, mais frequente entre os indivíduos de fototipos III à V que vivem em áreas onde há maior incidência dos raios ultravioletas (UV). 
No Brasil por apresentar grande miscigenação e possuir clima tropical tende a favorecer o surgimento da doença. O melasma não possui causa especifica, sua etiologia é multifatorial como: exposição solar, gravidez, uso de contraceptivo oral, alimentação, uso de cosméticos, procedimentos e processos inflamatórios dentre outros.

A exposição solar é o fator mais importante no surgimento do melasma. A radição UV interfere diretamente na melanogênese, que desencadeia na epiderme uma pigmentação mais escura. Gerando uma discromia, que se torna mais evidente nas áreas atingidas pelo melasma do que na pele adjacente. (GUINOT et al, 2010).

A hiperpigmentação melanogênica tende a melhorar no inverno e piorar consideravelmente no verão (ou a exposição intensa ao sol). O uso de protetor solar de alta proteção reduz até a metade o aparecimento do melasma e diminui sua incidência em até $90 \%$ na gravidez. (LAKHDAR et al, 2007)

Os hormônios sexuais como estrogênio e progesterona também estão relacionados ao surgimento do melasma, a gravidez e a terapia de reposição hormonal são fatores desencadeantes dessa disfunção (WV et al,2012)

A microcorrente parece viável para tratar a hiperpigmentação. Consiste numa corrente de baixa intensidade, subsensorial na faixa dos microampères, assemelhando-se a carga elétrica endógena, reconhecida como corrente fisiológica (BORGES, 2010).

Conforme Carvalho (2011), os efeitos desta microcorrente parecem relacionados à estimulação de microcirculação cutânea. Resultando desta forma, uma melhor oxigenação e nutrição tecidual. Daí a escolha deste tema. O presente artigo tem por objetivo: analisar os efeitos da microcorrente associada a vitamina $\mathrm{C}$ na hiperpigmentação facial.

O ácido ascórbico (vitamina C) tem a função de um antioxidante natural, que encontrase presente em alguns cítricos e vegetais verdes. Possui interação com íons de cobre no local ativo da tirosinase. Possui também atuação como agente redutor em diversas etapas oxidativas da formação da melanina. É um inibidor natural da melanogênese. O ácido ascórbico é um poderoso agente de ação clareadora (MONTEIRO et al, 2006).

Conforme Manela-Azulay et al (2003), é o ácido ascórbico indispensável na formação de fibras colágenas, que encontram-se presentes em praticamente todos os tecidos do corpo humano (seja derme, cartilagem ou ossos). Dentre os mais importantes benefícios descritos na literatura sobre a vitamina $\mathrm{C}$ tópica, destacam-se os seu efeitos: antioxidante, clareador e fotoprotetor, favorece também a hidratação da pele, além de estimular a produção de elastina e 
colágeno. Cabe destaque ao colágeno como ingrediente responsável pela elasticidade e resistência da pele, ou melhor, sem ele a pele torna-se mais flácida e frágil. Uma maior exposição a luz solar, parece um dos fatores que provocam alterações na síntese do colágeno, implicando na formação precoce de rugas e manchas na pele.

Diante dessas considerações, a utilização da vitamina C tópica demonstra-se viável já que funciona como um inibidor da melanogênese, atenuando o melasma. Para uma melhor aparência estética da pele, é necessário maior rigor do profissional, quando da realização de procedimentos, para que sejam seguros e que venham proporcionar os resultados adequados ao caso de cada cliente.

É uma vitamina hidrossolúvel de grande importâncoa para a saúde, embora não seja sintetizada pelo organismo (SANTOS; OLIVEIRA, 2014).

Segundo Bergmann, et.al; 2015, a vitamina C evidencia efeitos importantes na pele, tais como o reparo e defesa contra os agentes nocivos, crescimento e maturação das células consequentemente ocasionando no clareamento de manchas na pele. $\mathrm{O}$ uso tópico da vitamina C é utilizado para prevenção de danos solares que acometem a pele como o melasma.

A vitamina $\mathrm{C}$ tópica em solução aquosa penetra facilmente na pele e fica acumulada na barreira subcutânea. A pele absorve até $15 \%$ do ácido ascórbico em até 48hs. Quando usada de forma tópica, a vitamina é acumulada na pele com níveis superiores aqueles conseguidos através da alimentação; portanto seu uso tem sido muito eficaz permitindo que o mesmo adquira suas funções na pele (DALCIN, et.al, 2003).

Segundo Cayne (2008) a vitamina C possui função antioxidante e também atua inibindo o efeito da enzima tirosinase, permitindo que haja uma descoloração, sua ação antioxidante tem como princípio proteger a pele contra os efeitos nocivos dos radicais livres. O seu uso tópico aumenta significativamente o aspecto e a textura da epiderme, melhorando a elasticidade do tecido.

Entre suas variadas funções, a vitamina $\mathrm{C}$ tem uma atuação na fase aquosa, como um potente antioxidante sobre os radicais livres. Dessa forma, ela participa do sistema de proteção antioxidante (CATANIA; BARROS; FERREIRA, 2009; PENTEADO, 2003).

O papel da vitamina $\mathrm{C}$ no tecido conjuntivo tem sido reconhecido há bastante tempo. Trata-se de uma vitamina essencial para o funcionamento das células, além de seu aproveitamento pelo tecido conjuntivo durante a formação do colágeno, pois ela é um cofator fundamental (MANELA-AZULAY et al., 2003; STRUTZEL et al., 2007). 


\section{Considerações Finais}

Partindo do principal objetivo deste artigo, no qual foi compor mediante base na literatura cientifica sobre o referido tema, comparando diversos autores e corroborando por meio do processo de metanálise. Obteve-se como resultado desta proposta através de pesquisas recentes que o melasma pode ser controlado com técnicas menos agressivas. A vitamina $\mathrm{C}$ (ácido ascórbico), vem sendo muito utilizada na produção de cosméticos e produtos dermatológicos em geral, por apresentar importantes benéficos efeitos fisiológicos na pele. Seu potencial como antioxidante é amplamente reconhecido no meio científico.

Os resultados da ação da vitamina $\mathrm{C}$, em uso tópico, em manchas na pele, parece ter sua ação comprovada. Produtos que use em sua composição a vitamina $\mathrm{C}$, têm mostrado efeitos benéficos em tratamentos deste tipo. Pode-se afirmar dessa forma, que a vitamina $C$, é um produto com múltiplas funções, já que implica em variadas ações, tal como potente antioxidante, proporcionando a estimulação da eliminação de radicais livres, retardando o processo de envelhecimento da pele; Protetor do DNA das células, minimizando a ação das radiações UVA, que aceleram a predisposição ao câncer de pele e aumento das rugas; Ação clareadora, inibindo a tirosinase, evitando a formação de manchas, agindo como um protetor da pele em qualquer estação do ano.

Os efeitos da microcorrente na hiperpigmentação, podem reduzir a coloração escura causada pelo acúmulo de melanina isso devido aos efeitos que a microcorrente causa no tecido conjuntivo como: Aumento do ATP, agindo nas funções dos fibroblastos (aumentando a quantidade de colágeno e fibras elásticas), estímulo do processo de reparo e regeneração tecidual, normalização do PH local. Promovendo assim a revitalização e o rejuvenescimento da pele. Não há relatos na literatura em relação à utilização da microcorrente na hiperpigmentação, porém Soriano et al. (BORGES, 2006) citam o efeito de microestimulação produzido pela Microcorrente, gerando um importante estímulo da microcirculação cutânea com consequente melhora da nutrição celular, portanto tem uma influência na hiperpigmentação.

É importante ressaltar que, não há cura definitiva para o melasma, porém o tratamento combinado com ácido ascóbico e microcorrente são eficazes devido as suas ações fisiológicas.

Este estudo tem como objetivo sistematizar a eficácia dos tratamentos menos invasivos no controle do melasma, corroborando para que o profissional de estética e áreas afins possam 
explorar cada vez mais técnicas indolores e menos agressivas para tratar as disfunções estéticas, bem como descartar métodos que venham agravar ou mesmo comprometer a estética do mesmo.

\section{Referências}

ADALATKHAN, H., AMANI, F. A correlação entre melasma, cistos ovarianos e hormônios androgênicos (estudo de controle de casos). Res Biol Sci. 2007; 2: 593-6.

BOLANCA I, BOLANCA Z, KUNA K, VUKOVIC A, TUCKAR N, HERMAN R, et al. Chloasma-the mask of pregnancy. Coll Antropol. 2008;32:139-41.

GIL, ANTONIO CARLOS. Como elaborar projetos de pesquisa. 4. ed. São Paulo: Atlas, 2008.

GUINOT C, CHEFFAI S, LATREILLE J, DHAOUI MA, YOUSSEF S, JABER K, et al. Aggravating factors for melasma: a prospective study in 197 Tunisian patients. J Eur Acad Dermatol Venereol. 2010;24:1060-9.

HANDEL, ANA CAROLINA; MIOT, LUCIANE DONIDA BARTOLI; MIOT, HÉLIO AMANTE. Melasma: uma revisão clínica e epidemiológica. A. Bras. Dermatol., Rio de Janeiro, v. 89, n. 5, p. 771-782, outubro de 2014.

HALDER RM, GRIMES PE, MCLAURIN CI, KRESS MA, KENNEY JA JR. Incidence of common dermatoses in a predominantly black dermatologic practice. Cutis. 1983;32:388,390.

LAKHDAR H, ZOUHAIR K, KHADIR K, ESSARI A, RICHARD A, SEITÉ S, et al . Avaliação da eficácia de um protetor solar de amplo espectro na prevenção do chloasma em mulheres grávidas. J Eur Acad Dermatol Venereol. 2007; 21: 738

SANCHEZ NP, PATHAK MA, SATO S, FITZPATRICK TB, SANCHEZ JL, MIHM MC JR. Melasma: a clinical, light microscopic, ultrastructural, and immunofluorescence study. J Am Acad Dermatol. 1981;4:698-710.

TRAYNOR BJ, BRUIJN L, CONWIT R, BEAL F, O'NEILL G, FAGAN SC, et al. Neuroprotective agents for clinical trials in ALS: systemic assessment.Neurology. 2006; 67(1): 20-7.

VARANI J, DAME MK, RITTIE L, FLIGIEL SE, KANG S, FISHER GJ, et al. Decreased.collagen production in chronologically aged skin: Roles of age-depen-dent alteration in fibroblast function and defective mechanical stimula-tion. Am J Pathol.2006;168(6):18618 . 
WV IB, LAMBERT C, LOTTI TM, HERCOGOVÁ J, SINTIM-DAMOA A, SCHWARTZ RA. Melasma. G Ital Dermatol Venereol. 2012;147:413-8.

\section{Como citar este artigo (Formato ABNT):}

SILVA, Adriana da; PINHEIRO, Lívia Mara G. Ácido Ascórbico e Eletroterapia - Terapia Combinada no Tratamento do Melasma: Uma Revisão da Literatura. Id on Line Rev.Mult. Psic., 2018, vol.12, n.40, p.639-649. ISSN: 1981-1179.

Recebido: 07/05/2018

Aceito 11/05/2018 